\title{
BJMHR
}

British Journal of Medical and Health Research

Journal home page: www.bjmhr.com

\section{Dental Medicine Students and the Usage of Additional Supplements for Better Oral Hygiene}

\section{Petrovski Mihajlo*, Terzieva-Petrovska Olivera, Carceva-Salja Sofija,Ristova Ivana Faculty of medical sciences, University "Goce Delcev" - Stip, Macedonia}

\begin{abstract}
With adequate oral health knowledge, dental medicine students can act as role models for their patients in improving the oral health condition for the general population. We know that except using dental brush and tooth paste there are a lot of other additional supplements. The aim of this research was to assess the usage of these additional supplements among the students of dental medicine. For realizing the aim a questionnaire with adequate questions was made. The questionnaire was answered by total number of 137 dental medicine students at our university. Results showed that $74.25 \%$ from the students use additional supplements. Most of them, $66.26 \%$ use dental floss, $16.12 \%$ of them use interdental brushes, and just 8.2 $\%$ use both of them. $67.64 \%$ from the students are using mouthwash solutions. Most of the students that use mouthwash solution also use interdental brushes or dental floss. We can conclude that more than a two-third of the students are using additional supplements for maintaining adequate oral hygiene. This is very important because most of them will be dental professionals in the future, so they can help educating their patients maintaining their adequate oral hygiene
\end{abstract}

Keywords: Dental floss, Dental students, Oral health, Oral hygiene. 


\section{INTRODUCTION}

Dental plaque is a colorless, opalescent, slimy structure attached on the teeth, but also everywhere in the oral cavity: on crowns, dental bridges and dentures. Depending on the location there are three types of plaque: oral, gingival and dental plaque. Dental plaque is most important because of his role in the etiology of periodontal disease and dental caries. ${ }^{1}$ The best way to define dental plaque as a community of microorganisms found on a tooth surface as a biofilm, embedded in a matrix of polymers of host and bacterial origin. ${ }^{2}$

Plaque forms in an ordered way and has a diverse microbial composition that in healthy conditions remains relatively stable over time (microbial homeostasis). The predominant species from diseased sites are different from those found in healthy sites, although the putative pathogens can often be detected in low numbers at normal sites. ${ }^{3}$

Biofilms have been found to be involved in a wide variety of microbial infections (by one estimate $80 \%$ of all infections). These include dental caries, periodontal disease, otitis media, musculosceletal infections, necrotizing fasciitis, biliary tract infection, osteomyelitis, bacterial prostatitis, native valve endocarditis, meloidosis, cystic fibrosis pneumonia and periimplantitis. Salient features of these infections are persistence and chronicity. ${ }^{4}$

That's the reason why controlling or removing the dental plaque is the most important part of the oral hygiene.

Because the role of microorganisms in the causation and pathogenesis of periodontal disease is well documented, therapy is directed primarily towards reducing the number of pathogenic microorganisms in contact with periodontal tissues. Therefore, mechanical plaque removal is the basis of most periodontal treatment regimens.

If comparative analysis of the mechanical ways for removing of the dental plaque would be best to classify them into the following three groups:

\section{Tooth brushes}

-manual

-electrical

\section{Interdental cleaning aids}

-Dental floss

-wooden tips

-period-aid

-interdental brushes

-Rubber tip

Oral irrigation devices 5 
The effective removing to the dental plaque can be completed with using some chemical methods of plaque control as:

1. Enzymes : mucinase

2. Antibiotics: penicillin, vancomycin, erythromycin

3. Phenols: thymol

4. Quaternary ammonium compounds: benzankonium chloride, cetylpyridinum chloride

5. Bisbiguanides: chlorhexidine

Bispyridines: octenidine ${ }^{6}$

As mentioned, removing the dental plaque is the most important part of the oral hygiene. Using only dental brush and toothpaste doesn't allow complete removal of dental plaque, especially to the inteproximal spaces. Inadequate cleaning of these surfaces may cause aproximal caries, inflammatory gingival hyperplasia, periodontal pockets formation etc. This is the reason why additional supplements for cleaning dental plaque from interproximal space should be used. Many problems begin in the interproximal spaces. This is the main purpose of interdental brush - to clean where standard toothbrushes can't.

Mouthwash solutions usually contain more than one active ingredient and can show more indications. Mouthwash solutions are used as a part of home care for special needs such as control of the biofilm, caries prevention or reduction of infection. Their importance is big especially in individuals with certain problems due to the fact they are not being able to brush or mechanically remove dental plaque. However, mechanical removal of plaque is the most important procedure.

The importance of using additional supplements for oral hygiene is guiding and motivates us to do a study among students in our faculty whether they pay attention to good oral hygiene because they will be future professionals who need to raise awareness of oral hygiene for patients.

Behavior and attitude of oral health providers, especially dentists, toward oral health affect their capacity to deliver oral health care services. The aim of this research was to assess the usage of these additional supplements among the students of dental medicine at University "Goce Delcev" - Stip.

\section{MATERIALS AND METHOD}

All 137 students of Dental medicine at University "Goce Delcev" - Stip were included in this research. Each of the students have received and filled a questionnaire about their own oral hygiene. For easier statistical processing of the data in the questionnaire we had used 
questions with offered answers. Presented data originates from research conducted over a period from 15 September 2016 to 1 of October 2016.

Questions from the questionnaire were:

1) Do you use dental floss?

a) Yes

b) No

2) If the answer is yes, how often?

a) Everyday

b) Once a week

c) Twice a week

d) Once for a month

3) Do you use interdental brushes?

a) Yes

b) $\mathrm{No}$

4) Do you use mouth rinse solution?

a) Yes

b) No

5) What kind of mouth rinse solution do you use?

a) Alcoholic

b) Non-alcoholic

6) How do you think that studying dental medicine influenced to your oral health and hygiene?

Data were aggregated, while the values are presented in accordance with the recommendations of the World Health Organization expressed by numerical values expressed values to two decimal places. Results of the survey were processed in two ways manually and using specialized computer software-Statistica 9 for Windows and Microsoft Excel 2012.

The processed data are presented in tables and graphs using as needed. The results obtained in the test population are represented in percentage.

\section{RESULTS AND DISCUSSION}

After processing the results we have noted that $74.25 \%$ from the student use additional supplements. Most of them, $66.26 \%$ use dental floss, $16.12 \%$ of them use interdental brushes, and just $8.2 \%$ use both of them. $67.64 \%$ from the students uses mouthwash solutions, most of them non-alcoholic. Also, most of the students that use mouthwash solution also use interdental brushes or dental floss. (Figure 1, Figure 2, Figure 3, Figure 4.) 


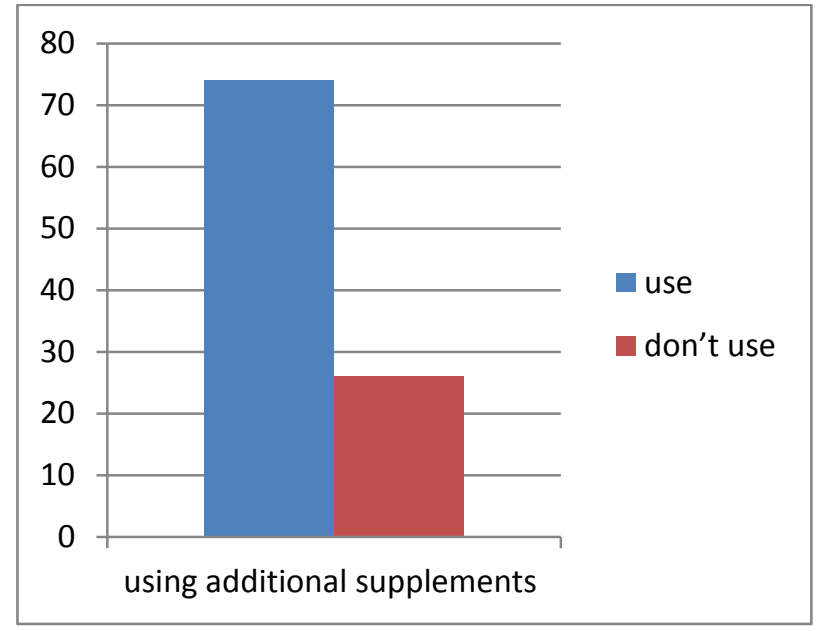

Figure 1: Usage of additional supplements for oral hygiene among dental medicine students

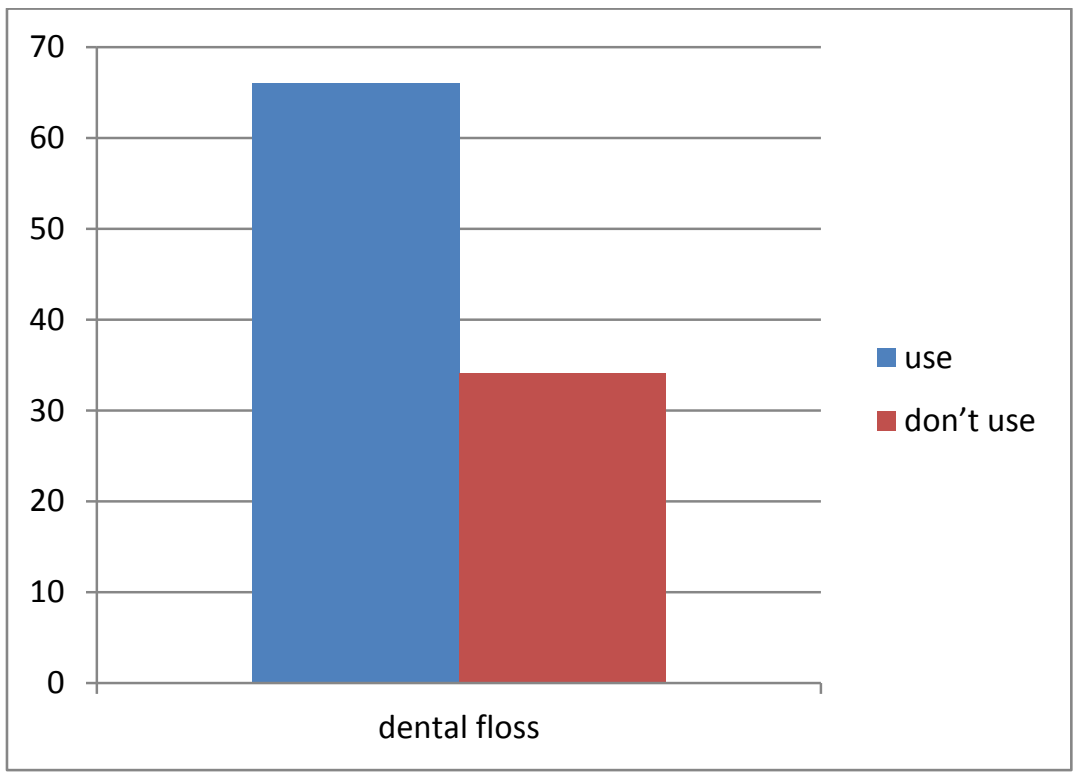

Figure 2: Usage of dental floss among dental medicine students

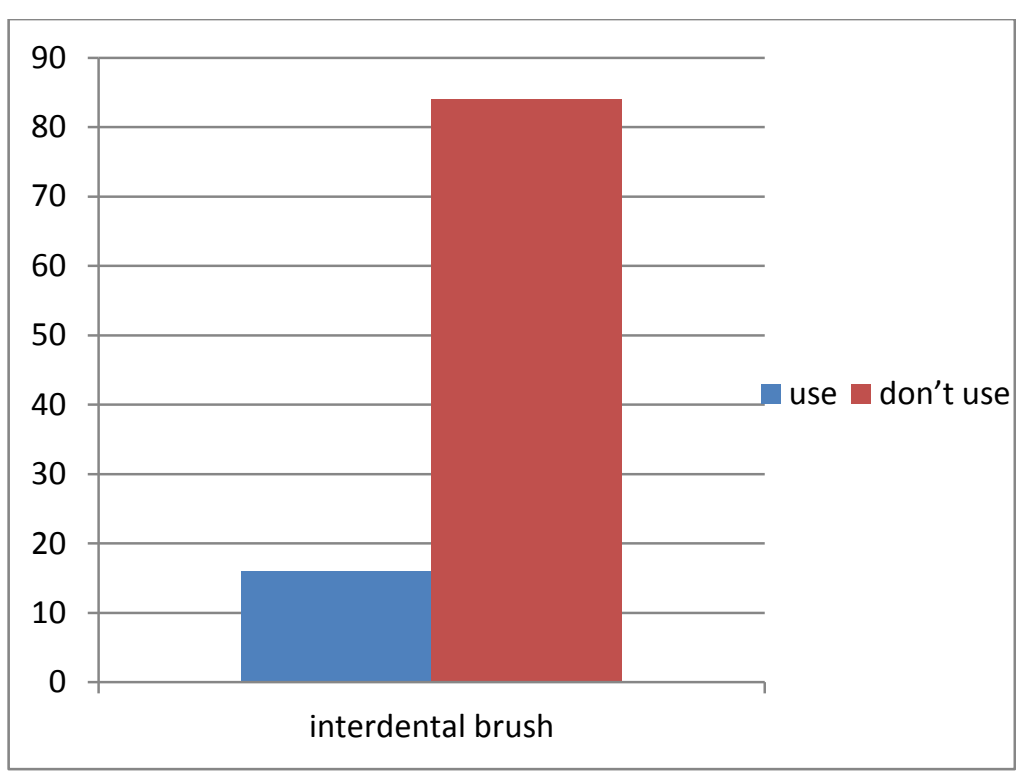

Figure 3: Usage of interdental brushes among dental medicine students 


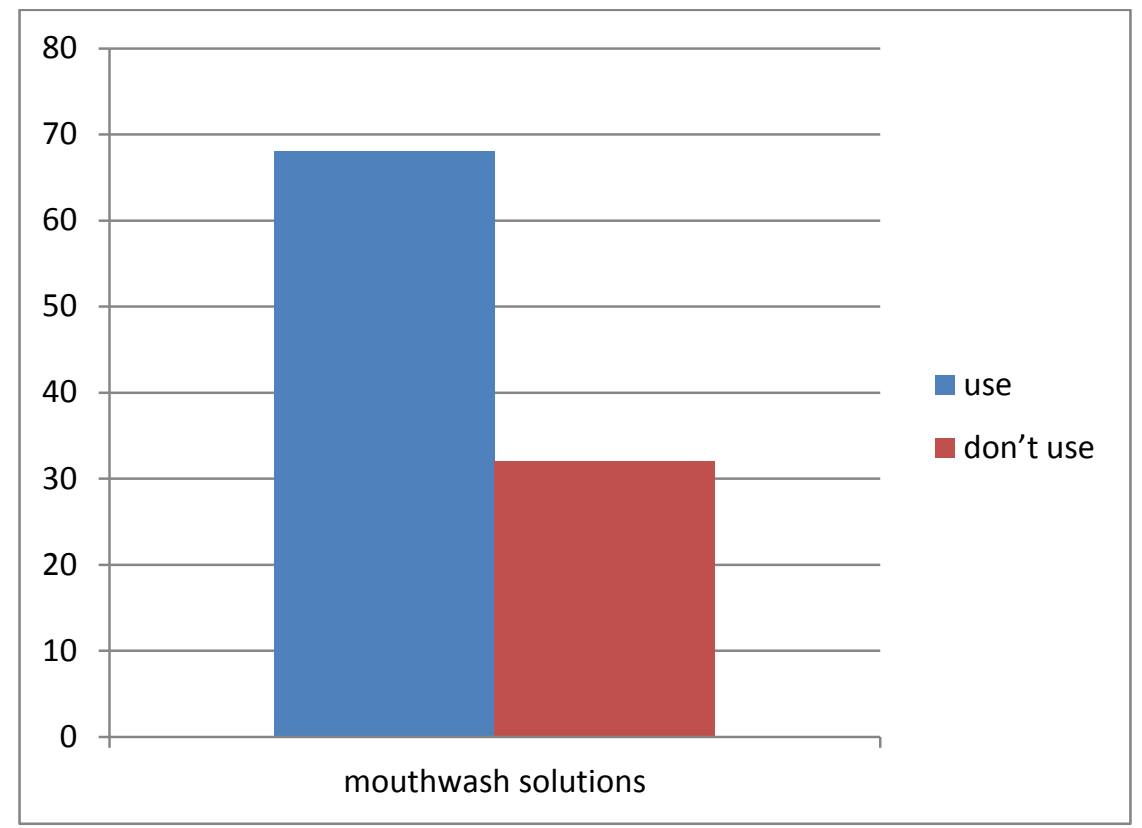

Figure 4: Usage of mouth washing solutions among dental medicine students

And the most important question of all, how you feel that your study so far contributed to the improvement of oral hygiene? According to the results it can be noted that in over $80 \%$ studying of dental medicine have a positive impact on oral hygiene (more precisely $81.5 \%$ ). Also $79 \%$ of the respondents have a subjective feeling that adequately remove dental plaque from their teeth. (Figure. 5)

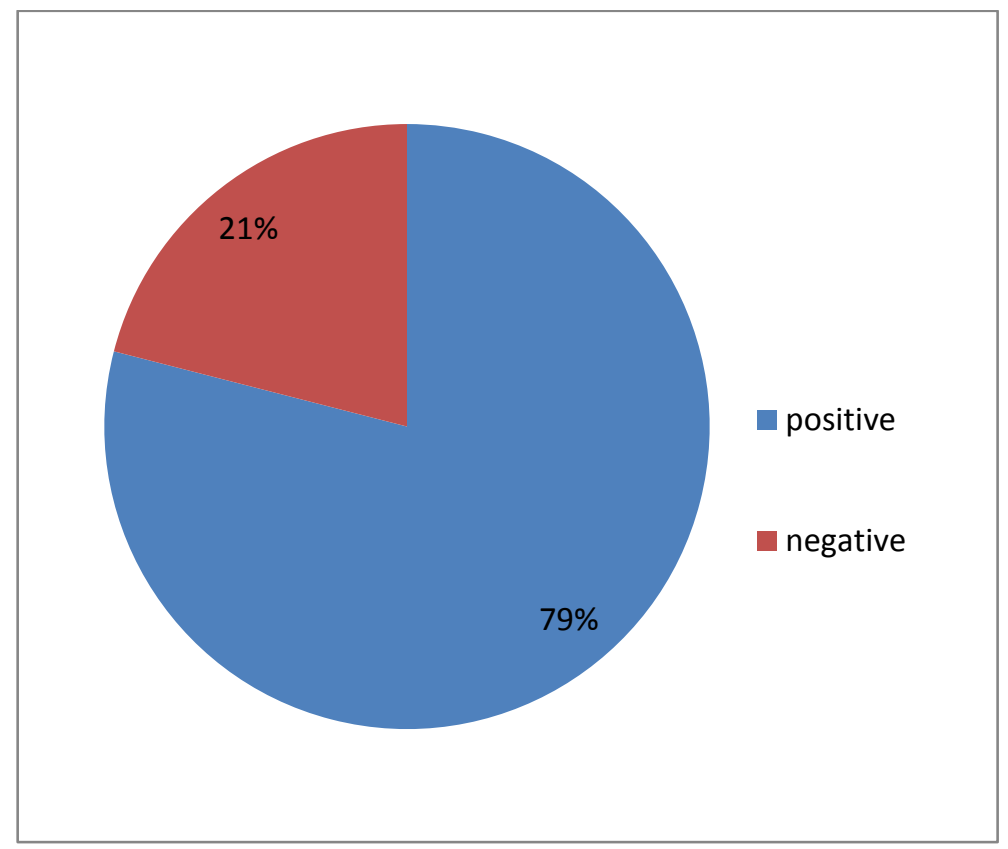

Figure 5: Impact of the dental medicine studies on improving of the oral hygiene among dental medicine students

\section{DISCUSSION}

Dental medicine students, as future professionals, specialized for treatment of oral diseases as well as in preventive services, should have a good oral health knowledge and attitude. With 
appropriate and adequate oral health knowledge and behavior, they can act as models for patients and play a positive role in improving oral health condition of the general population. Trough the dental medicine studies students teach many academic subjects which influence of their knowledge and behavior toward oral health. ${ }^{7}$

In order to perfectly perform their future health mission of preventing and treatment of oral diseases, dental medicine students should have good and evidence-based knowledge regarding educating their patients about oral health behaviors and habits.

The removal of interproximal plaque is considered to be important for the maintenance of gingival health, prevention of periodontal disease and the reduction of caries. Unfortunately, the toothbrush is relatively ineffective at removing interproximal plaque, and therefore patients need to resort to additional techniques. ${ }^{8}$

However, the habits and attitudes of dental students towards usage of additional supplements have not been investigated in our country and around so far.

University students are in a dynamic transition period of growth and development that bridges adolescence and adulthood. ${ }^{9}$ That is why in this period may occur a very destructive behavior and manifestations that can have a negative effect of oral health.

Better oral health is in correlation with gingival health not only among university students. Improving self-efficacy and usage of additional supplements may be beneficial for maintaining good gingival health. According to Muzitani at all, ${ }^{10}$ to prevent gingivitis, the approach of enhancing self-efficacy of oral hygiene among university students can be useful. According to the results obtained and presented in our study may be noted that $3 / 4$ of the students, or $74.25 \%$ of subjects use additional supplements for maintaining of adequate oral hygiene. There is a high level of satisfaction with this high rate of dental students who use the additional funds for oral hygiene.

Dental flosss is the most widely used method of interdental cleaning. The most widely used method to evaluate interproximal contacts is to pass dental floss between contact areas. ${ }^{8}$ Ideal interproximal contacts between adjacent natural teeth or dental restorations are a critical factor for the health of the periodontium and dento-alveolar complex.

Results from our research showed a high percentage of students $(66.26 \%)$ using dental floss as a method for removing interdental plaque. Satisfactorily high percentage of students who use dental floss greatly indicates the high percentage of persons performing interproximal cleaning.

Patients, as students of dentistry found flossing difficult, especially where there are tight contact points, and therefore interdental cleaning does not readily become an established part 
of daily oral hygiene. That is why, there is a need for new techniques, methods and devices to be developed so interdental cleaning can become easier and improve patient motivation.

As interdental cleaning with dental floss can be effective but it is techniquesensitive. Interdental brushes have been shown to be more effective compared to floss in plaque index scores, but not in gingival inflammation reduction. ${ }^{11}$

Results of our research show that a very small percentage $(16.62 \%)$ of the students are using interdental brushes. Therefore there is a need for education and prevention programs in the context of increased use of interdental brushes.

Dental floss has been used for many years in conjunction with tooth brushing for removing dental plaque in interdental spaces, however, interdental brushes are easier to use than floss, providing there is sufficient space between the teeth. ${ }^{12}$ Interdental brush are used as an adjunct to tooth brushing removes more dental plaque than brushing alone, being even more effective than dental floss or wood sticks in removing dental plaque. ${ }^{13}$ According to Slot et all there is a positive significant difference using interdental brushes with respect to the plaque scores, bleeding scores and probing pocket depth. ${ }^{14}$

Incorporating a mouth rinse solution with normal tooth brushing could be a useful adjunct to oral hygiene. ${ }^{15}$ The chemical control of plaque includes organic or inorganic chemicals, which inhibit the accumulation, growth and survival of microbiota and debris. ${ }^{16}$

A variety of products are available chemical plaque control, which are divided into first generation (e.g., phenols, quaternary ammonium compounds), second generation (e.g., bisbiguanides-chlorhexidine gluconate $[\mathrm{CHXG}]$ ), and third generation (e.g. delmopinol). ${ }^{17}$ CHXG is widely used with concentration of as mouth rinse.

In our research, a high percentage of subjects use mouthwash solution, more precisely $67.64 \%$. Most of them use non-alcoholic mouthwash solution. Uses of mouthwash in conjunction with mechanical aids are effective in reducing plaque and gingival scores. ${ }^{18}$

There is a difference in the relation between oral health and hygiene among students of students of dental medicine and students of other faculties. Munz et all ${ }^{19}$ showed that the medical students had lower oral health-related and less positive attitudes about oral healthrelated behavior than the dental hygiene and dental students. According to them, third- and fourth-year dental students' knowledge, skills and behavior were higher than those of firstand second-year students. Polychronopoulou et all ${ }^{20}$ also showed differences in the oral health behavior between dental students in preclinical as opposed to clinical semesters, because according to them, comprehension for the subject and profession develops mainly during the clinical part of the study. 
Dental medicine students from the first couple of years have limited knowledge of oral health and lack serious attitude toward oral cleaning behaviors. In one research in Romania, it is noted that students are defective with interproximal cleaning procedures. Floss, wood sticks, rubber tips and interdental brushes currently represent the primary methods available for interproximal cleaning. ${ }^{21}$

The task of spreading this awareness needs to be extended trough the professional students. ${ }^{22}$ Perhaps the most significant results presented in this study refer to the impact of studying dentistry on interproximal cleaning and oral hygiene. $81.5 \%$ from the student of dental medicine have subjective filling that there is a positive impact on oral hygiene due to dental medicine studying. Also $79 \%$ of the respondents have a subjective feeling that adequately remove dental plaque from all surfaces of their teeth.

As future leaders in oral health care, dental students have a major role in patient education and oral health promotion. It is expected that dental students will become exemplars in their own oral health attitudes and behaviors, and these may reflect the manner in which their future roles in oral health promotion and education are fulfilled. Therefore, acquiring knowledge and attitudes related to dental health and the prevention of oral diseases is very important during the future dentists' training period. ${ }^{23}$

Oral health is an essential component of general health and quality of life. The relevance of oral health lies in the relationship between the effective oral health knowledge and the behavior. As dentists, in the future we have to reinforcing the importance of correcting all aspects related with brushing and flossing along with the importance of regular checkups. ${ }^{22}$ Because, little is known about the oral health behaviors and attitudes of dental students and the influence of educational training on the development of their oral behaviors and attitudes we hope that this research will become an incentive for further research on this topic.

\section{CONCLUSION}

We can conclude that more than a two-third of the students use additional supplements for maintaining adequate oral hygiene. That is very important because most of them will be dental professionals in future, so they can help in education of the patients for maintain adequate oral hygiene. But the low percentage of students that use interdental brushes has to be improved. Dental education experience definitely improved the health attitudes and behavior with more positive attitudes found during the $4^{\text {th }}$ and $5^{\text {th }}$ year of dental education.

\section{ACKNOWLEDGMENTS}

We would like warmly to thank to all dental medicine students at University "Goce Delcev"Stip, who participated in this research. 


\section{REFERENCES}

1. Nakova, M., Ivanovski, K., Pesevska, S. 2003. Basics of oral physiology and biochemistry. Faculty of dentistry, Skopje [macedonian] : 113

2. Marsh, P.D. 2004. Dental plaque as a microbial biofilm. Caries Res, 38 : 204-211.

3. Marsh, PD. 2006. Dental plaque as a biofilm and a microbial community implications for health and disease. BMC Oral Health, 6(Suppl 1): S14

4. Socransky, S.S., Haffajee, AD. 2002. Dental biofilms: difficult therapeutic targets. Periodontol. 2000, 28: 12-55

5. Petersilka, G.J., Ehmke, B., Flemmig, T.F. 2008.Antimicrobial effects of mechanical debridement. Periodontol. 2000, 28: 56-71.

6. Chandki, R., Banthia, P., Banthia, R. 2011. Biofilms: A microbial home. Journal of Indian Society of Periodontology, 15(2), 111-114.

7. Halboub, E.S., Al-Maweri, S.A., Al-Jamaei, A.A., Al-Wesabi, M.A., Shamala, A., Al-Kamel, A., Alsharani, A., Eissa, N. 2016. Self-Reported Oral Health Attitudes and Behavior of Dental and Medical students, Yemen. Glob J Health Sci. 8(10):56676. doi: 10.5539/ gjhs.v8n10p143.

8. Warren, P.R, Chater, B.V. 1996. An overview of established interdental cleaning methods.J Clin Dent. 7(3 Spec No):65-9.

9. Kojima, A. 2017. Relationships between Self-Rated Oral Health, Subjective Symptoms, Oral Health Behavior and Clinical Conditions in Japanese University Students: A Cross-Sectional Survey at Okayama University. BMC Oral Health, 13: 62.

10. Mizutani, S., Ekuni, D., Furuta, M., Tomofuji, T., Irie, K., Azuma, T., Kojima, A., Nagase, J., Iwasaki, Y., Morita, M. 2012. Effects of self-efficacy on oral health behaviours and gingival health in university students aged 18- or 19-years-old. J Clin Periodontol. 39(9):844-9. doi: 10.1111/j.1600051X.2012.01919.x. Epub 2012 Jul 11.

11. Teich, S.T., Joseph, J., Sartori, N., Heima, M., Duarte, S. 2014. Dental floss selection and its impact on evaluation of interproximal contacts in licensure exams. J Dent Educ. 78(6):921-6

12. Poklepovic, T., Worthington, H.V., Johnson, T.M., Sambunjak, D., Imai, P., Clarkson, J.E., Tugwell, P. 2013. Interdental brushing for the prevention and control of periodontal diseases and dental caries in adults. Cochrane Database Syst Rev.18;(12):CD009857. doi: 10.1002/14651858.CD009857.pub2. 
13. Rasines, G. 2009. The use of interdental brushes along with toothbrushing removes most plaque. Evid Based Dent. 10(3):74. doi: 10.1038/sj.ebd. 6400666.

14. Slot, D.E., Dörfer, C.E., Van der Weijden, G.A. 2008. The efficacy of interdental brushes on plaque and parameters of periodontal inflammation: a systematic review. Int $J$ Dent Hyg. 6(4):253-64. doi: 10.1111/j.16015037.2008.00330.x.

15. Cantore, S., Ballini, A., Morim, G., Dibello, V., Marrelli, M., Mirgaldi, R., De Vito, D., Tatullo, M. 2016. Anti-plaque and antimicrobial efficiency of different oral rinses in a 3-day plaque accumulation model. J Biol Regul Homeost Agents. 30(4):1173-1178.

16. Prasad, K. A., Varma, R. 2015. Anti-Plaque Efficacy of Herbal and $0.2 \%$ Chlorhexidine Gluconate Mouthwash: A Comparative Study.Journal of International Oral Health : 7.8: 98-102.

17. Mhaske, M., Samad, B.N., Jawade, R., Bhansali, A. 2012. Chemical agents in control of dental plaque in dentistry: An overview of current knowledge and future challenges. Adv Appl Sci Res, 3(1):268-72.

18. Godha, S., Dasar, P. L., Sandesh, N., Mishra, P., Kumar, S., Balsaraf, S., Vyas, S. 2016. Impact of different oral hygiene aids for the reduction of morning bad breath among dental students: a crossover clinical trial. Clujul Medical, 89(4), $525-533$

19. Munz, S.M., Kim, R.Y., Holley, T.J., Donkersloot, J.N., Inglehart, M.R.2017. Dental Hygiene, Dental, and Medical Students' OMFS/Hospital Dentistry-Related Knowledge/Skills, Attitudes, and Behavior: An Exploration. $J$ Dent Educ. 81(2):149-161

20. Polychronopoulou, A., Kawamura, M., Athanasouli,T. 2002. Oral self-care behavior among dental school students in Greece. J Oral Sci 44: 73-78

21. Dumitrescu, A.Z., Kawamura, M., Dogaru, B.A., Dogaru, C.D. 2010. Relation of Achievement Motives, Satisfaction with Life, Happiness and Oral Health in Romanian University Students. Oral Health Prev Dent 8; 1: 15-22, doi:10.3290/j.ohpd.a18807

22. Dayakar, M. M., Kumar, J., Pai, G. P., Shivananda, H., \& Rekha, R. 2016. A survey about awareness of periodontal health among the students of professional colleges in Dakshina Kannada District. Journal of Indian Society of Periodontology, 20(1), 67-71. http://doi.org/10.4103/0972-124X.168487 
23. Lalani, A., Dasar, P.L., Sandesh, N., Mishra, P., Kumar, S., Balsaraf, S. 2015. Assessment of relationship between oral health behavior, oral hygiene and gingival status of dental students. Indian J Dent Res;26:592-7

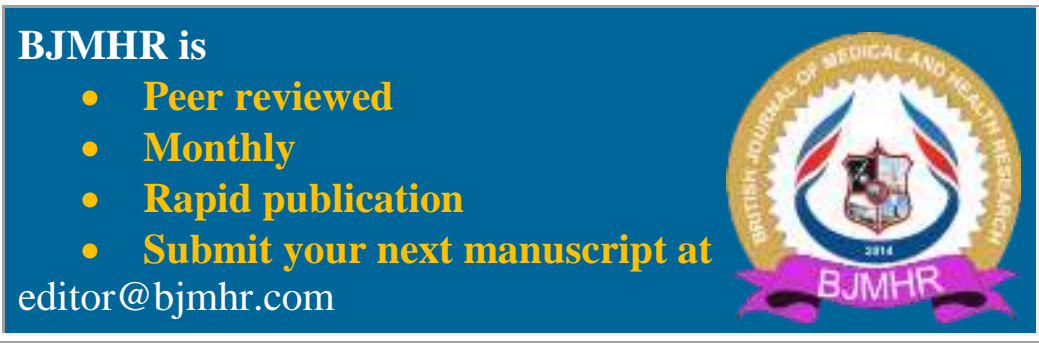

